Math. Res. Lett. 15 (2008), no. 4, 613-622

\title{
THE SHARP CONSTANT IN THE HARDY-SOBOLEV-MAZ'YA INEQUALITY IN THE THREE DIMENSIONAL UPPER HALF-SPACE
}

\author{
Rafael D. Benguria, Rupert L. Frank, and Michael Loss
}

\begin{abstract}
It is shown that the sharp constant in the Hardy-Sobolev-Maz'ya inequality on the upper half space $\mathbb{H}^{3} \subset \mathbb{R}^{3}$ is given by the Sobolev constant. This is achieved by a duality argument relating the problem to a Hardy-Littlewood-Sobolev type inequality whose sharp constant is determined as well.
\end{abstract}

\section{Introduction}

The present work is concerned with a particular case of the Hardy-Sobolev-Maz'ya inequality

$$
\int_{\mathbb{H}^{n}}\left[|\nabla f(\mathbf{x})|^{2}-\frac{1}{4 y^{2}}|f(\mathbf{x})|^{2}\right] d \mathbf{x} \geq C_{n}\left(\int_{\mathbb{H}^{n}}|f(\mathbf{x})|^{\frac{2 n}{n-2}} d \mathbf{x}\right)^{\frac{n-2}{n}} .
$$

Here $f$ is a function that lives in the half space

$$
\mathbb{H}^{n}:=\left\{\mathbf{x}=(x, y): x \in \mathbb{R}^{n-1}, y>0\right\}
$$

in a sense to be defined later. For the moment the reader may assume that $f$ is compactly supported in $\mathbb{H}^{n}$. It is quite easy to see that the left side of (1) is positive; this is Hardy's inequality. That (1) holds for a strictly positive constant $C_{n}$ if $n \geq 3$ was proved by Maz'ya [9] (Section 2.1.6., Corollary 3). In what follows, $C_{n}$ denotes the sharp constant in the above inequality. It was shown in recent work by Tertikas and Tintarev [10] among other things, that an optimizer for the sharp constant $C_{n}$ exists provided the dimension $n \geq 4$. Using a trial function they show that $C_{n}<S_{n}$ where $S_{n}$ is the Sobolev constant and then use a concentration compactness argument to establish the existence of an optimizer. Except for the fact that $C_{n}<S_{n}$ for $n \geq 4$ nothing is known about the value of $C_{n}$.

The inequality (1) has a number of equivalent formulations. For once it is equivalent to the inequality

$$
\int_{\mathbb{B}^{n}}|\nabla g(\boldsymbol{\Omega})|^{2} d \boldsymbol{\Omega}-\int_{\mathbb{B}^{n}} \frac{1}{\left(1-|\boldsymbol{\Omega}|^{2}\right)^{2}}|g(\boldsymbol{\Omega})|^{2} d \boldsymbol{\Omega} \geq C_{n}\left(\int_{\mathbb{B}^{n}}|g(\boldsymbol{\Omega})|^{\frac{2 n}{n-2}} d \boldsymbol{\Omega}\right)^{\frac{n-2}{n}}
$$

where $\mathbb{B}^{n}$ is the unit ball in $\mathbb{R}^{n}$. To see this, set

$$
f(x, y)=\left(\frac{2}{(1+y)^{2}+x^{2}}\right)^{\frac{n-2}{2}} g(B(x, y)),
$$

Received by the editors May 12, 2007.

(C) 2007 by the authors. This paper may be reproduced, in its entirety, for non-commercial purposes. 
where $B$ is the Möbius transformation that maps the upper half space $\mathbb{H}^{n}$ to the unit ball $\mathbb{B}^{n}$, i.e.,

$$
\boldsymbol{\Omega}=B(x, y)=\frac{\left(2 x, 1-x^{2}-y^{2}\right)}{(1+y)^{2}+x^{2}} .
$$

Inserting (4) into (1), a straightforward computation involving some integration by parts yields (3). Clearly, this inequality is invariant under rotations. Note that these two representations, the one on the half space and the one on the unit ball show the invariance of the inequality under all Möbius transformations that preserve the upper half space. By restricting these transformations to the bounding hyperplane one obtains the Möbius group of $\mathbb{R}^{n-1}$, i.e., the group generated by the Euclidean transformations that preserve this hyperplane together with scaling and inversion about the unit sphere. Thus, the Möbius group that preserves the upper half space and the Möbius group of $\mathbb{R}^{n-1}$ are isomorphic. This indicates that the term containing the expression $\left(1-|\boldsymbol{\Omega}|^{2}\right)^{-2}$ has some intrinsic geometric meaning.

Another natural way to rewrite the problem (1) is via stereographic projection from the unit ball to the hyperboloid $\mathbb{P}^{n}$. Once more, set

$$
g(\boldsymbol{\Omega})=\left(\frac{2}{1-|\boldsymbol{\Omega}|^{2}}\right)^{\frac{n-2}{2}} k(P(\mathbf{u}))
$$

where

$$
P(\mathbf{u})=\frac{\left(2 \Omega, 1+|\Omega|^{2}\right)}{1-|\Omega|^{2}} .
$$

It is easy to check that $P$ maps the unit ball to the upper branch of the hyperboloid $u^{2}-v^{2}=-1$, where $\mathbf{u}=(u, v), u \in \mathbb{R}^{n}$ and $v \in \mathbb{R}$. Inserting (6) into (3) yields the equivalent inequality

$$
\int_{\mathbb{P}^{n}}|\nabla k(\mathbf{u})|^{2} d \mathrm{Vol}-\frac{(n-1)^{2}}{4} \int_{\mathbb{P}^{n}}|k(\mathbf{u})|^{2} d \mathrm{Vol} \geq C_{n}\left(\int_{\mathbb{P}^{n}}|k(\mathbf{u})|^{\frac{2 n}{n-2}} d \mathrm{Vol}\right)^{\frac{n-2}{n}}
$$

The metric used here on $\mathbb{P}^{n}$ is the one induced by the Euclidean space $\mathbb{R}^{n+1}$. Note that $(n-1)^{2} / 4$ is the infimum of the spectrum of the hyperbolic Laplacian $-\Delta_{\mathbb{P} n}$.

As mentioned before, the half space problem has been investigated in [10], but in its formulation on the hyperbolic space it has also been investigated before (see [6] and references in there) although under a different point of view. There, one asks whether there exists a constant $B_{n}$ such that the inequality

$$
\int_{\mathbb{P}^{n}}|\nabla k(\mathbf{u})|^{2} d \mathrm{Vol} \geq S_{n}\left(\int_{\mathbb{P}^{n}}|k(\mathbf{u})|^{\frac{2 n}{n-2}} d \mathrm{Vol}\right)^{\frac{n-2}{n}}+B_{n} \int_{\mathbb{P}^{n}}|k(\mathbf{u})|^{2} d \mathrm{Vol}
$$

holds. Here, $S_{n}$ is the Sobolev constant,

$$
\frac{n(n-2)}{4}\left|\mathbb{S}^{n}\right|^{\frac{2}{n}}
$$

where $\left|\mathbb{S}^{n}\right|$ is the volume of the $n$-dimensional unit sphere in $\mathbb{R}^{n+1}$. For $n>3$ the sharp constant $B_{n}=\frac{n(n-2)}{4}$ (see [6]). Note that $\frac{n(n-2)}{4}<\frac{(n-1)^{2}}{4}$. In this language, the problem investigated in [10] is different, i.e., replace $B_{n}$ by the optimal constant 
and then find the sharp constant $C_{n}$ that will replace $S_{n}$. Certainly $C_{n} \leq S_{n}$, in fact $C_{n}<S_{n}$ for $n>3$. Note that, in this case the exact value of $C_{n}$ is not known.

In both formulations the interesting case $n=3$ is conspicuously absent and it is this case we would like to address in this letter. Before stating the theorem we mention some preliminaries. For smooth functions of compact support on $\mathbb{H}^{n}$ one has that

$$
\int_{\mathbb{H}^{n}}|\nabla f(x, y)|^{2} d x d y-\int_{\mathbb{H}^{n}} \frac{f(x, y)^{2}}{4 y^{2}} d x d y=\int_{\mathbb{H}^{n}} y|\nabla g(x, y)|^{2} d x d y
$$

where

$$
g(x, y)=\frac{f(x, y)}{\sqrt{y}} .
$$

Since the right side of (11) is positive, we can extend it to all functions $f$ for which this expression is finite. More precisely we say that $f \in D_{1}\left(\mathbb{H}^{n}\right)$ if the corresponding $g$ vanishes at infinity in $d x y d y$ - measure, and the weak derivative of $g(x, y)$ is squareintegrable with respect to this measure. Recall that 'vanishing at infinity in $d x y d y$ measure' means that the set $\{|g(x, y)|>a\}$ has finite $d x y d y$ - measure for every $a>0$. Note, that for such functions, the two terms in (1) may be separately infinity, but their difference is well defined and finite. For such functions it was established in [10] that for $n \geq 4$ an optimizer exists for (1).

We have

THEOREM 1.1. Let $f \in D_{1}\left(\mathbb{H}^{3}\right)$. Then $f \in L^{6}\left(\mathbb{H}^{3}\right)$ and the inequality

$$
\int_{\mathbb{H}^{3}}|\nabla f(\mathbf{x})|^{2} d \mathbf{x} \geq \int_{\mathbb{H}^{3}} \frac{1}{4 y^{2}}|f(\mathbf{x})|^{2} d \mathbf{x}+S_{3}\left(\int_{\mathbb{H}^{3}}|f(\mathbf{x})|^{6} d \mathbf{x}\right)^{\frac{1}{3}}
$$

holds where $S_{3}$ is the sharp Sobolev constant in three dimensions, i.e.,

$$
S_{3}=3(\pi / 2)^{4 / 3} .
$$

The inequality is always strict for nonzero f's. Using the formulation on hyperbolic space we have the inequality

$$
\int_{\mathbb{P}^{3}}|\nabla k(\mathbf{u})|^{2} d \mathrm{Vol} \geq S_{3}\left(\int_{\mathbb{P}^{3}}|k(\mathbf{u})|^{6} d \mathrm{Vol}\right)^{\frac{1}{3}}+\int_{\mathbb{P}^{3}}|k(\mathbf{u})|^{2} d \mathrm{Vol} .
$$

At first sight (13) seems to contradict the well known fact that Hardy's inequality

$$
\int_{\mathbb{H}^{3}}|\nabla f(\mathbf{x})|^{2} d \mathbf{x} \geq \int_{\mathbb{H}^{3}} \frac{1}{4 y^{2}}|f(\mathbf{x})|^{2} d \mathbf{x}
$$

as well as Sobolev's inequality

$$
\int_{\mathbb{H}^{3}}|\nabla f(\mathbf{x})|^{2} d \mathbf{x} \geq S_{3}\left(\int_{\mathbb{H}^{3}}|f(\mathbf{x})|^{6} d \mathbf{x}\right)^{\frac{1}{3}}
$$

are sharp in the sense that in each the constant on the right side cannot be replaced by a larger one. None of them, however, has a non-zero optimizer and the optimizing sequence in Hardy's inequality are far from optimal for Sobolev's inequality and vice versa. In fact Theorem 1.1 says that if $h_{m}, m=1,2, \ldots$ is an optimizing sequence for Hardy's inequality, then necessarily $\left\|h_{m}\right\|_{6} \rightarrow 0$ as $m \rightarrow \infty$ and likewise if $g_{m}$ is 
an optimizing sequence for Sobolev's inequality on the half-space we necessarily must have that $\int g_{m}^{2} / y^{2} \rightarrow 0$ as $m \rightarrow \infty$.

Inequality (15) which is equivalent to (13) shows the remarkable fact that in three dimensions $-\Delta_{\mathbb{P}^{3}}-1$, as far as Sobolev's inequality is concerned, behaves as $-\Delta$ on $\mathbb{R}^{3}$.

In contrast to the case $n=3$, for $n \geq 4$ the sharp constant is always attained for some nonzero function (see [10]).

The problem (1) has been generalized to the case where the underlying domain $D$ is a convex set. In this case one replaces $\frac{1}{4 y^{2}}$ by $\frac{1}{4 d(x)^{2}}$ where $d(x)$ is the distance of the point $x \in D$ to the boundary of $D$. It is conjectured in [10] that the sharp constant for convex domains is given by the half space problem. This is true for the case where the domain is a ball. First we define $D_{1}\left(\mathbb{B}^{n}\right)$ to be those functions $g$ for which the corresponding function $f$ given by $(4)$ is in $D_{1}\left(\mathbb{H}^{n}\right)$. We have

THEOREM 1.2. Let $g \in D_{1}\left(\mathbb{B}^{n}\right)$. Then $g \in L^{\frac{2 n}{n-2}}\left(\mathbb{B}^{n}\right)$ and

$$
\int_{\mathbb{B}^{n}}|\nabla g(\boldsymbol{\Omega})|^{2} d \boldsymbol{\Omega}-\int_{\mathbb{B}^{n}} \frac{1}{4(1-|\boldsymbol{\Omega}|)^{2}}|g(\boldsymbol{\Omega})|^{2} d \boldsymbol{\Omega} \geq C_{n}\left(\int_{\mathbb{B}^{n}}|g(\boldsymbol{\Omega})|^{\frac{2 n}{n-2}} d \boldsymbol{\Omega}\right)^{\frac{n-2}{n}} .
$$

For nonzero $g$ 's the inequality is always strict.

The inequality follows directly from (3) by noting that for $|\boldsymbol{\Omega}|<1$,

$$
\frac{1}{\left(1-|\boldsymbol{\Omega}|^{2}\right)^{2}}>\frac{1}{4(1-|\boldsymbol{\Omega}|)^{2}} \text {. }
$$

That the inequality is sharp and always strict for non-zero functions can be seen by scaling down a compactly supported 'almost' optimizer of the half space problem and use this as a trial function for the ball problem. Note that this device also works for general convex domains. The hard part is to establish the analog of (18) for general convex domains.

An amusing consequence of the formulation (3) is that by inversion with respect to the unit sphere one obtains a sharp inequality on the complement of the unit ball, i.e., we have

THEOREM 1.3. The inequality

$$
\int_{\left(\mathbb{B}^{n}\right)^{c}}|\nabla g(\boldsymbol{\Omega})|^{2} d \boldsymbol{\Omega}-\int_{\left(\mathbb{B}^{n}\right)^{c}} \frac{1}{\left(1-|\boldsymbol{\Omega}|^{2}\right)^{2}}|g(\boldsymbol{\Omega})|^{2} d \boldsymbol{\Omega} \geq C_{n}\left(\int_{\left(\mathbb{B}^{n}\right)^{c}}|g(\boldsymbol{\Omega})|^{\frac{2 n}{n-2}} d \boldsymbol{\Omega}\right)^{\frac{n-2}{n}}
$$

holds for all functions $g$ with the property that $|x|^{-(n-2)} g\left(\frac{x}{|x|^{2}}\right) \in D_{1}\left(\mathbb{B}^{n}\right)$. Moreover, for $n>3$ equality can be attained.

The appropriate formulation of this inequality for general domains, not necessarily convex, is an open problem. Theorem 1.3 suggests that the 'correct' inequality is formulated in terms of either the harmonic radius or the hyperbolic radius of a domain $D$. For a definition of these concepts we refer the reader to [1]. Both of these objects are conformally covariant, i.e., under conformal transformations they scale with the $n$-th root of the Jacobian. In the case of a ball, the two concepts coincide and are equal to $\left(1-|\boldsymbol{\Omega}|^{2}\right)$. Since the ball and the half space are conformally the same, these 
two concepts coincide also on the half space and are given by $2 y$. Thus, it is natural to ask for which domain $D$ does the inequality

$$
\int_{D}\left[|\nabla f|^{2}-\frac{1}{R(x)^{2}}|f(x)|^{2}\right] d^{n} x \geq C_{n}\left(\int_{D}|f(x)|^{\frac{2 n}{n-2}} d^{n} x\right)^{\frac{n-2}{n}}
$$

hold? Here $R(x)$ is either the harmonic radius or the hyperbolic radius. While the sharp constant $C_{n}$ is the same for conformally equivalent domains, is it the same for all convex domains? We do not know the answers to these questions.

The plan of the paper is the following. In Section 2 we derive the Green function for fractional powers of the operator $-\Delta-\frac{1}{4 y^{2}}$. This yields Hardy-Littlewood-Sobolev type kernels. In Section 3 we prove $L^{p}$ estimates for these kernels and recover Theorem 1.1 .

\section{The Green function}

From now on the dimension $n$ is arbitrary. It is convenient to start with the following heat type equation on the upper half space $\mathbb{H}^{n}$

$$
u_{t}=\Delta u+\frac{1}{4 y^{2}} u, u(x, y ; 0)=f(x, y) .
$$

Substituting $u=\sqrt{y} g$ one obtains the equation

$$
g_{t}=\Delta_{x} g+g_{y y}+\frac{1}{y} g_{y}, g(x, y ; 0)=\frac{f(x, y)}{\sqrt{y}},
$$

and one sees that the right side of the equation is an $n+1$ dimensional Laplacian. Note that $g_{y y}+\frac{1}{y} g_{y}$ is the two dimensional Laplacian of a radial function. A similar idea has been used in [2] in a different context. With this in mind one arrives at once at the following formula for the solution of the heat equation

$$
u(x, y ; t)=\int_{\mathbb{H}^{n}} G\left(x-x^{\prime}, y, y^{\prime} ; t\right) f\left(x^{\prime}, y^{\prime}\right) d x^{\prime} d y^{\prime}
$$

where

$$
G\left(x-x^{\prime}, y, y^{\prime} ; t\right)=\left(\frac{1}{4 \pi t}\right)^{\frac{n+1}{2}} \sqrt{y y^{\prime}} e^{-\frac{\left(x-x^{\prime}\right)^{2}+y^{2}+y^{\prime 2}}{4 t}} \int_{0}^{2 \pi} e^{\frac{y y^{\prime}}{2 t} \cos \phi} d \phi .
$$

It is not hard to see that this heat kernel is a contraction semigroup on $L^{2}\left(\mathbb{H}^{n}\right)$ with Lebesgue measure. Thus, the generator $Q$ is a selfadjoint operator and it is an extension of $-\Delta-\frac{1}{4 y^{2}}$ originally defined on smooth functions with compact support in $\mathbb{H}^{n}$. Note that the $L^{2}$-norm of the gradient of functions in the domain of $Q$ is in general not finite. We shall continue to use the symbol $-\Delta-\frac{1}{4 y^{2}}$ to denote $Q$.

It is straight forward to see (see e.g., Theorem 7.10 in [8]) that

$$
\lim _{t \rightarrow 0} \frac{1}{t}\left[\|f\|_{L^{2}\left(\mathbb{H}^{n}\right)}^{2}-\left(f, G_{t} f\right)_{L^{2}\left(\mathbb{H}^{n}\right)}\right]=2 \pi \int_{\mathbb{H}^{n}}\left(\left|\nabla_{x} g\right|^{2}+\left|g_{y}\right|^{2}\right) y d y d x
$$

where $G_{t} f$ is the solution of the intial value problem (22) and $g=\frac{f}{\sqrt{y}}$. Note that the right hand side is manifestly positive and coincides with the interpretation of $-\Delta-\frac{1}{4 y^{2}}$ given in [10]. 
Via the heat kernel we find the kernel of the fractional powers

$$
\left(-\Delta-\frac{1}{4 y^{2}}\right)^{-\frac{\alpha}{2}}\left(\mathbf{x} ; \mathbf{x}^{\prime}\right)=\frac{1}{\Gamma\left(\frac{\alpha}{2}\right)} \int_{0}^{\infty} t^{\frac{\alpha}{2}} G\left(x-x^{\prime}, y, y^{\prime} ; t\right) \frac{d t}{t},
$$

for $\alpha>0$, and a calculation leads to the expression

$$
\begin{aligned}
& \left(-\Delta-\frac{1}{4 y^{2}}\right)^{-\frac{\alpha}{2}}\left(\mathbf{x} ; \mathbf{x}^{\prime}\right) \\
= & 2^{-\alpha} \pi^{-\frac{n+1}{2}} \frac{\Gamma\left(\frac{n+1-\alpha}{2}\right)}{\Gamma\left(\frac{\alpha}{2}\right)} \sqrt{y y^{\prime}} \int_{0}^{2 \pi}\left[\left(x-x^{\prime}\right)^{2}+y^{2}+y^{\prime 2}-2 y y^{\prime} \cos \phi\right]^{-\frac{n+1-\alpha}{2}} d \phi \\
=: & \Phi_{n, \alpha}\left(\mathbf{x} ; \mathbf{x}^{\prime}\right) .
\end{aligned}
$$

These formulae hold provided that $0<\alpha<n+1$.

Similarly, for $0<\alpha<n$ well known expressions hold for $(-\Delta)^{-\frac{\alpha}{2}}$ on $\mathbb{R}^{n}$ which, for reasons that become clear later, we write in terms of the variables $(x, y)$ as

$$
\begin{aligned}
& (-\Delta)^{-\frac{\alpha}{2}}\left(\mathbf{x} ; \mathbf{x}^{\prime}\right) \\
= & 2^{-\alpha} \pi^{-\frac{n}{2}} \frac{\Gamma\left(\frac{n-\alpha}{2}\right)}{\Gamma\left(\frac{\alpha}{2}\right)}\left[\left(x-x^{\prime}\right)^{2}+\left(y-y^{\prime}\right)^{2}\right]^{-\frac{n-\alpha}{2}} \\
= & : \quad \Gamma\left(\frac{n-\alpha}{2}\right) \Psi_{n, \alpha}\left(\mathbf{x} ; \mathbf{x}^{\prime}\right) .
\end{aligned}
$$

Note that $\Psi_{n, \alpha}$ is also defined for $n=\alpha$. First we state some simple pointwise properties about the kernel $\Phi_{n, \alpha}$.

LEMMA 2.1. If $n \leq \alpha<n+1$, we have that

$$
\sup _{a} \Phi_{n, \alpha}\left(x, y+a ; x^{\prime}, y^{\prime}+a\right)=\lim _{a \rightarrow \infty} \Phi_{n, \alpha}\left(x, y+a ; x^{\prime}, y^{\prime}+a\right) \equiv \infty .
$$

If $n-1 \leq \alpha<n$ for $n \geq 2$ and $0<\alpha<1$ for $n=1$, we have that

$$
\sup _{a} \Phi_{n, \alpha}\left(x, y+a ; x^{\prime}, y^{\prime}+a\right)=\lim _{a \rightarrow \infty} \Phi_{n, \alpha}\left(x, y+a ; x^{\prime}, y^{\prime}+a\right) \equiv \Psi_{n, \alpha}\left(\mathbf{x} ; \mathbf{x}^{\prime}\right) \text {. }
$$

In this case

$$
\Phi_{n, \alpha}\left(\mathbf{x} ; \mathbf{x}^{\prime}\right)=\Psi_{n, \alpha}\left(\mathbf{x}, \mathbf{x}^{\prime}\right) F(A)
$$

where

$$
A=\frac{\sqrt{y y^{\prime}}}{\left|\mathbf{x}-\mathbf{x}^{\prime}\right|}
$$

and $F(A)$ is strictly increasing towards $\Gamma\left(\frac{n-\alpha}{2}\right)$.

Proof. An elementary calculation shows that (32) holds for all $\alpha<n+1$ with $F$ defined as

$$
F(A)=\frac{\Gamma\left(\frac{n+1-\alpha}{2}\right)}{\sqrt{\pi}} \int_{-\pi}^{\pi} \frac{A}{\left[1+2 A^{2}(1-\cos (\phi))\right]^{\frac{n+1-\alpha}{2}}} d \phi .
$$

All the statements are an immediate consequence of Lemma 4.1 with $\beta=\frac{n+1-\alpha}{2}$. 


\section{3. $L^{p}$-estimates for fractional powers}

As a consequence of Lemma 2.1 and Lieb's sharp constant in the Hardy-Littlewood Sobolev inequality [7] we have the following corollary.

COROLLARY 3.1. If $n \leq \alpha<n+1$ then the operator

$$
\left(-\Delta-\frac{1}{4 y^{2}}\right)^{-\frac{\alpha}{2}}
$$

is not bounded from $L^{p}\left(\mathbb{H}^{n}\right)$ to $L^{q}\left(\mathbb{H}^{n}\right)$ for any $1 \leq p, q \leq \infty$. If $n \geq 2$ and $n-1 \leq$ $\alpha<n$ (resp. $0<\alpha<1$ if $n=1$ ), then this operator is a bounded operator from $L^{p}\left(\mathbb{H}^{n}\right)$ to $L^{q}\left(\mathbb{H}^{n}\right)$ for all $1<p, q<\infty$ that satisfy

$$
\frac{1}{q}=\frac{1}{p}-\frac{\alpha}{n}
$$

and its norm coincides with the one of $(-\Delta)^{-\alpha / 2}: L^{p}\left(\mathbb{R}^{n}\right) \rightarrow L^{q}\left(\mathbb{R}^{n}\right)$.

Moreover, for such values of $\alpha$ we have

$$
\left(f,\left(-\Delta-\frac{1}{4 y^{2}}\right)^{-\frac{\alpha}{2}} f\right) \leq 2^{-\alpha} \pi^{-\frac{n}{2}} \frac{\Gamma\left(\frac{n-\alpha}{2}\right)}{\Gamma\left(\frac{\alpha}{2}\right)} C(n, \alpha)\|f\|_{p}^{2}
$$

where $p=\frac{2 n}{n+\alpha}$ and

$$
C(n, \alpha)=\pi^{\frac{n-\alpha}{2}} \frac{\Gamma\left(\frac{\alpha}{2}\right)}{\Gamma\left(\frac{n+\alpha}{2}\right)}\left[\frac{\Gamma\left(\frac{n}{2}\right)}{\Gamma(n)}\right]^{-\frac{\alpha}{n}}
$$

is the sharp constant. This constant is not attained in (37) for nonzero functions.

Note that the inequality

$$
\int_{\mathbb{R}^{n} \times \mathbb{R}^{n}} f(\mathbf{x}) g(\mathbf{y}) \Phi_{n, \alpha}(\mathbf{x}, \mathbf{y}) d \mathbf{x} d \mathbf{y} \leq \int_{\mathbb{R}^{n} \times \mathbb{R}^{n}} f(\mathbf{x}) g(\mathbf{y}) \Psi_{n, \alpha}(\mathbf{x}, \mathbf{y}) d \mathbf{x} d \mathbf{y}
$$

is always strict for non-negative functions $f, g$ which do not vanish identically. This follows from the fact that $\Phi_{n, \alpha}(\mathbf{x}, \mathbf{y})<\Psi_{n, \alpha}(\mathbf{x}, \mathbf{y})$ in $\mathbb{R}^{n} \times \mathbb{R}^{n}$ except on the diagonal $\mathbf{x}=\mathbf{y}$.

Proof of Theorem 1.1. Recall that $Q$ is a self-adjoint operator on $L^{2}\left(\mathbb{H}^{3}\right)$. Thus for $f \in L^{2}\left(\mathbb{H}^{3}\right)$ with $Q^{\alpha / 4} f \in L^{2}\left(\mathbb{H}^{3}\right)$ we have that

$$
|(f, h)|=\left|\left(Q^{\alpha / 4} f, Q^{-\alpha / 4} h\right)\right| \leq\left(f, Q^{\alpha / 2} f\right)^{1 / 2}\left(h, Q^{-\alpha / 2} h\right)^{1 / 2}
$$

which by Corollary 3.1 yields the bound

$$
|(f, h)|^{2} \leq 2^{-\alpha} \pi^{-\frac{n}{2}} \frac{\Gamma\left(\frac{n-\alpha}{2}\right)}{\Gamma\left(\frac{\alpha}{2}\right)} C(n, \alpha)\left(f, Q^{\alpha / 2} f\right)\|h\|_{p}^{2}
$$

for $n-1 \leq \alpha<n$ and $p=\frac{2 n}{n+\alpha}$. Thus, $f \in L^{p^{\prime}}\left(\mathbb{H}^{3}\right)$ with $p^{\prime}=\frac{2 n}{n-\alpha}$ and

$$
\|f\|_{p^{\prime}}^{2} \leq 2^{-\alpha} \pi^{-\frac{n}{2}} \frac{\Gamma\left(\frac{n-\alpha}{2}\right)}{\Gamma\left(\frac{\alpha}{2}\right)} C(n, \alpha)\left(f, Q^{\alpha / 2} f\right) .
$$


Under the assumption that $f \in L^{2}\left(\mathbb{H}^{3}\right)$, (13) follows by choosing $n=3$ and $\alpha=2$. To obtain the statement for $f \in D_{1}\left(\mathbb{H}^{3}\right)$ we can assume that $f=: \sqrt{y} g \geq 0$ and set $f_{C}=\sqrt{y} g_{C}$ where

$$
g_{C}(x, y)=\min \left(C,\left[g(x, y)-\frac{1}{C}\right]_{+}\right),
$$

$C$ is some large positive constant and $[x]_{+}:=\max (x, 0)$. Since $g$ vanishes at infinity in $d x y d y$ - measure,

$$
\int_{\mathbb{H}^{3}} g_{C}(x, y)^{2} d x y d y=\int_{\mathbb{H}^{3}} f_{C}(x, y)^{2} d x d y
$$

is finite.

Moreover,

$$
\int_{\mathbb{H}^{3}}\left|\nabla g_{C}(x, y)\right|^{2} d x y d y=\int_{1 / C \leq g \leq C+1 / C}|\nabla g(x, y)|^{2} d x y d y,
$$

and hence $Q^{1 / 2} f_{C} \in L^{2}\left(\mathbb{H}^{3}\right)$. Thus the inequality (13) holds for $f_{C}$. Now we let $C \rightarrow \infty$ and conclude, using (45) and (13), that $\left\|f_{C}\right\|_{6}$ is uniformly bounded. Using monotone convergence we find that $\lim _{C \rightarrow \infty}\left\|f_{C}\right\|_{6}=\|f\|_{6}<\infty$.

Assume that there is equality in (13) for some nonzero function $f \in D_{1}\left(\mathbb{H}^{3}\right)$. Then with $h=f^{p^{\prime}-1} /\|f\|_{p^{\prime}}^{p^{\prime}-1}$ we must have equality in (37) with $f$ replaced by $h$. This cannot be unless $h$ and hence $f$ vanish identically.

The reader may wonder what happens when $0<\alpha<n-1$. In this case the sharp constant in the inequality corresponding to (37) is not known and neither is the existence of an optimizer. The problem, however, is conformally invariant and in a similar fashion as in the Hardy-Sobolev-Maz'ya inequality, it can be transformed into one in the unit ball that is rotationally invariant. The device of competing symmetries developed in [4] allows to restrict the maximization problem to radial functions on the ball. Likewise, it is also possible to show that every maximizer, provided it exists, is the conformal image of a radial function.

\section{Appendix}

In this appendix we collect some facts about the function

$$
F(A):=\frac{\Gamma(\beta)}{\sqrt{\pi}} \int_{-\pi}^{\pi} \frac{A}{\left(1+2 A^{2}(1-\cos (\phi))\right)^{\beta}} d \phi,
$$

where $\beta=\frac{n+1-\alpha}{2}$.

LEMMA 4.1. Depending on the value of $\beta$, the function $F(A)$ has the following asymptotics as $A \rightarrow \infty$.

a) If $0<\beta \leq \frac{1}{2}$ then $\lim _{A \rightarrow \infty} F(A)=\infty$.

b) If $\frac{1}{2}<\beta \leq 1$, then $F(A)$ is a strictly monotone increasing function and

$$
\lim _{A \rightarrow \infty} F(A)=\Gamma\left(\beta-\frac{1}{2}\right) .
$$


Proof. Since

$$
F(A)=\frac{\Gamma(\beta)}{\sqrt{\pi}} G(A)
$$

where

$$
G(A)=\int_{-\pi A}^{\pi A} \frac{1}{\left(1+2 A^{2}\left(1-\cos \left(\frac{\phi}{A}\right)\right)\right)^{\beta}} d \phi
$$

the limit of $F(A)$ as $A \rightarrow \infty$ is

$$
\frac{\Gamma(\beta)}{\sqrt{\pi}} \int_{-\infty}^{\infty} \frac{1}{\left(1+\phi^{2}\right)^{\beta}} d \phi=\Gamma\left(\beta-\frac{1}{2}\right)
$$

for $\beta>\frac{1}{2}$ and it is $+\infty$ for $\beta \leq \frac{1}{2}$. This proves a). To see that b) holds for $\beta=1$ one easily performs the $\phi$ integration and obtains

$$
G(A)=\frac{2 \pi A}{\sqrt{1+4 A^{2}}}
$$

which is obviously strictly increasing with $A$. For $\frac{1}{2}<\beta<1$ we use the formula

$$
\begin{gathered}
{\left[1+2 A^{2}(1-\cos \phi)\right]^{-\beta}} \\
=\frac{\sin (\pi \beta)}{\pi} \int_{0}^{\infty}\left[1+t+2 A^{2}(1-\cos \phi)\right]^{-1} t^{1-\beta} \frac{d t}{t} .
\end{gathered}
$$

Integrating with respect to $\phi$ yields

$$
G(A)=2 \sin (\pi \beta) \int_{0}^{\infty} \frac{A}{\sqrt{(1+t)^{2}+4(1+t) A^{2}}} t^{1-\beta} \frac{d t}{t} .
$$

Again, this function increases strictly with $A$.

\section{Acknowledgements}

The authors thank Professor Tintarev for pointing out an inconsistency in an earlier formulation of Theorem 1.1. This work was partially supported by Fondecyt (CHILE) projects 106-0651 and 706-0200, and CONICYT/PBCT Proyecto Anillo de Investigación en Ciencia y Tecnología ACT30/2006 (R.D.B.), by the Swedish Foundation for International Cooperation in Research and Higher Education (STINT) (R.L.F.) and by NSF-grant DMS-0600037 (M.L.)

\section{References}

[1] C. Bandle and M. Flucher, Harmonic radius and concentration of energy; hyperbolic radius and Liouville's equations $\Delta U=e^{U}$ and $\Delta U=U^{\frac{n+2}{n-2}}$, SIAM Review, 38 (1996), 191-238.

[2] W. Beckner, On the Grushin operator and hyperbolic symmetry, Proc. Amer. Math. Soc. 129 (2000), 1233-1246.

[3] H. Brézis and E. H. Lieb, A relation between pointwise convergence of functions and convergence of functionals, Proc. Amer. Math. Soc. 88 (1983), 486-490.

[4] E. A. Carlen and M. Loss, On the minimization of symmetric functionals, Rev. Math. Phys. 6 (1994), 1011-1032.

[5] S. Filippas, V. G. Maz'ya, and A. Tertikas, Sharp Hardy-Sobolev inequalities, C. R. Math. Acad. Sci. Paris 339 (2004), no. 7, 483-486. 
[6] E. Hebey, Nonlinear Analysis on Manifolds: Sobolev Spaces and Inequalities, Courant Lecture Notes in Mathematics Vol. 5, AMS, Providence, RI, 1999.

[7] E. H. Lieb, Sharp constants in the Hardy-Littlewood-Sobolev and related inequalities, Ann. Math. 118 (1983), 349-374.

[8] E. H. Lieb and M. Loss, Analysis, Graduate Studies in Mathematics Vol. 14, AMS, Providence, 2001.

[9] V. G. Maz'ya, Sobolev Spaces, Springer Verlag, Berlin New York, 1985.

[10] A. Tertikas and K. Tintarev, On existence of minimizers for the Hardy-Sobolev-Maz'ya inequality, Ann. Mat. Pura Appl. (4) 186 (2007), no. 4, 645-662.

Department of Physics, P. Universidad Católica de Chile, Casilla 306, Santiago 22, CHILE

E-mail address: rbenguri@fis.puc.cl

Matematiska institutionen, KTH Stockholm, 10044 Stockholm, Sweden., Address as of September 2007: Department of Mathematics, Princeton University, Princeton, NJ 08544

E-mail address: rupert@math.kth.se, rlfrank@math.princeton.edu

School of Mathematics, Georgia Tech, Atlanta, GA 30332

E-mail address: loss@math.gatech.edu 